Journal of The Electrochemical Society, 154 (1) G1-G5 (2007)

G1

\title{
(4HS) \\ Porosity Effects on Properties of Mesoporous Silica Low- $k$ Films Prepared Using Tetraethylorthosilicate with Different Templates
}

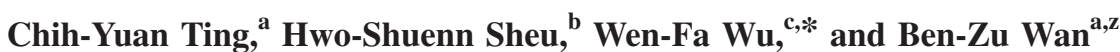

${ }^{a}$ Department of Chemical Engineering, National Taiwan University, Taipei 106, Taiwan

${ }^{b}$ National Synchrotron Radiation Research Center, Hsinchu 300, Taiwan

${ }^{c}$ National Nano Device Laboratories, Hsinchu 300, Taiwan

\begin{abstract}
A series of mesoporous silica films with different porosities and different pore sizes were prepared using different templates or different template concentrations in tetraethylorthosilicate colloid solutions. The amphiphilic templates such as cetyltrimethylammonium bromide $\left(\mathrm{C}_{16} \mathrm{TMABr}\right)$, Brij-56, Tween80, and Pluronic P123 were used. To investigate the effect of self-assembly surfactants on the film properties and to make a comparison, polyethylene glycol (molecular weight $=1450$ ) which is not a surfactant was also used as a template in this research. Various film properties such as porosity, refractive index, dielectric constant, leakage current density, flatband voltage, hardness, and Young's modulus were measured. For the film templated with $\mathrm{C}_{16} \mathrm{TMABr}$, the high leakage current density and positive flatband voltage were attributed to the residual bromide in the framework, which was from bromine in $\mathrm{C}_{16} \mathrm{TMABr}$ after the calcination process. Moreover, its abnormally low dielectric constant was mainly due to the high leakage current density. For the films made with nonionic templates, the dielectric constant and the refractive index can be reduced linearly by increasing the film porosities; in other words, they are strongly related to the porosity rather than the pore size or the ordering of pore structure. However, the larger leakage current density and the weaker mechanical strength for more porous films are the drawbacks. According to higher values of flatband voltage, the elevation of leakage current densities are attributed to more silanol groups in the films. It was shown that the films with more ordered structures or thicker pore wall possessed better mechanical strength. Nevertheless, the porosity is the most influential factor upon mechanical strength of the films.

(C) 2006 The Electrochemical Society. [DOI: 10.1149/1.2388852] All rights reserved.
\end{abstract}

Manuscript submitted May 10, 2006; revised manuscript received August 28, 2006. Available electronically November 21, 2006.

Mesoporous silica materials prepared by a surfactant-templated method have been attempted for a variety of applications. ${ }^{1-3}$ The advantage of the surfactant-templated method is its ability to create different pores with controllable sizes in silica. By introducing air in the pores, the dielectric constant and the refractive index of the materials can be reduced. The high porosities and the controllable pore sizes make this kind of material a candidate for future low- $k$ films or for future optical coatings. In the past few years, the films with low dielectric constant and the films that enhance transmittance have been successfully developed. ${ }^{4-10}$ However, the mechanical properties are especially important for the subsequent processes such as chemical mechanical polishing (CMP). ${ }^{11}$ Several attempts to improve the mechanical properties have been reported. ${ }^{12-15}$ Among them Cho et al. ${ }^{14}$ have recently developed a process using ozone ashing treatment. The leakage current of the material has to be low because the low dielectrics serve as an electric insulator in the device as well. For the mesoporous materials, all these properties should be related to the porosity of the film. To push the surfactanttemplated materials into real applications, knowing the correlations between the film porosity and various film properties is important. Nevertheless, related information about the subject is still fragmented. Recently, a systematic research about structural characterization of mesoporous organosilica films was reported. ${ }^{16}$ Only cetyltrimethylammonium bromide $\left(\mathrm{C}_{16} \mathrm{TMABr}\right)$, Brij-76, and Pluronic F127 were used as the templates. The dielectric constant $(k)$ and the refractive index $(n)$ were correlated with the film porosity. However, only Young's modulus (one of the mechanical properties) of the films prepared with $\mathrm{C}_{16} \mathrm{TMABr}$ template was presented, the values of which appeared low due to the mixture of tetraethylorthosilicate (TEOS) and methyltrimethoxy silane (MTMS) were used as the silica source. Moreover, the leakage current density and flatband voltage, which are important physical properties of low- $k$ films, were not mentioned. ${ }^{16}$ Therefore, in this research, in order to examine the correlations more comprehensively, the films with different porosities were prepared using different templates and different template concentrations in the colloids.To make the films with stronger

* Electrochemical Society Activity Member.

z E-mail: benzuwan@ntu.edu.tw mechanical strength, TEOS instead of the mixture of TEOS and MTMS was used as the only silica source in this research. A surface modification process was used to make the films hydrophobic. The amphiphilic templates investigated were $\mathrm{C}_{16} \mathrm{TMABr}$, Brij-56, Tween80, and Pluronic P123. Except $\mathrm{C}_{16} \mathrm{TMABr}$, they were different from those reported before. ${ }^{16}$ Moreover, to investigate the effect of self-assembly surfactants on the film properties and to make a comparison, polyethylene glycol (molecular weight $=1450$ ), which is not a surfactant, was also used as a template in this research. Various properties such as porosity, refractive index, dielectric constant, leakage current density, flatband voltage, hardness, and Young's modulus of each mesoporous film were measured. The effects of porosity are presented.

\section{Experimental}

Sample preparation.- The preparation of coating solution has been described previously. ${ }^{6,7}$ The coating solution was a mixture of TEOS, deionized water $\left(\mathrm{H}_{2} \mathrm{O}\right)$, ethanol $(\mathrm{EtOH})$, hydrochloric acid $(\mathrm{HCl})$, and templates. TEOS was from Merck, and the other chemicals were from Acros. The molar ratio of TEOS $/ E t O H / \mathrm{H}_{2} \mathrm{O} / \mathrm{HCl}$ in the coating solution was $1: 8: 4: 0.25$. If not specified, the weight ratio of template/TEOS was maintained at 0.41 . The coating solution was stirred at room temperature for $3 \mathrm{~h}$. The resulting solution was spincoated on a 4 in. p-type silicon $(1,0,0)$ wafer at $2600 \mathrm{rpm}$ for $30 \mathrm{~s}$ with an SSP-01A spinner (King Polytechnic Engineering Co.). After the spin coating, the film was baked at $106^{\circ} \mathrm{C}$ for $1 \mathrm{~h}$. The calcination was performed at $400^{\circ} \mathrm{C}$ (the temperature increasing rate was $1^{\circ} \mathrm{C} / \mathrm{min}$ ) for $0.5 \mathrm{~h}$ in the air with a type 48000 furnace (Barnstead|Thermolyne). The film surface was modified to be hydrophobic by immersing it in a hexamethyldisiloxane (HMDS)/ toluene solution at $80^{\circ} \mathrm{C}$ for $1.5 \mathrm{~h}$.

Characterization.-X-ray diffraction (XRD) and nitrogen adsorption/desorption measurements were used for the characterization of pore structure of the films. For the measurements, the calcined films were scraped off from 12 slices of 4 in. silicon wafers and collected together. XRD was carried out at the beamline 17A of the National Synchrotron Radiation Research Center (NSRRC) in Taiwan. The wavelength of the X-ray was $0.1326 \mathrm{~nm}$. Nitrogen adsorption/desorption isotherms were obtained at $77 \mathrm{~K}$ from a Mi- 


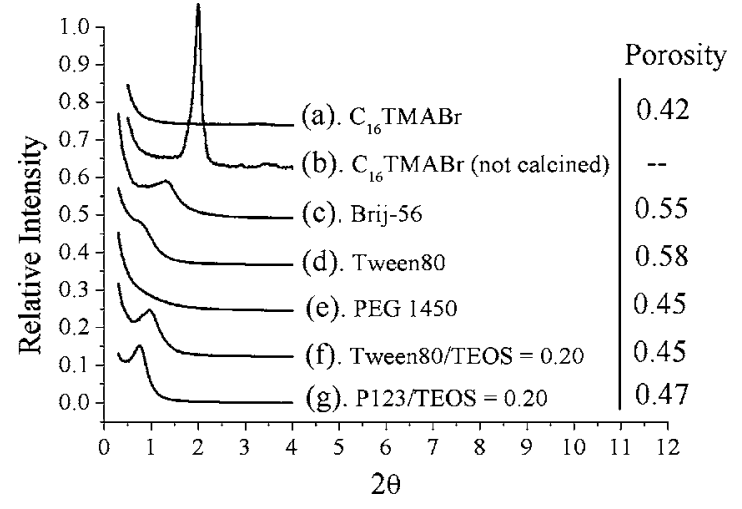

Figure 1. XRD patterns and porosities of the films studied in this research.

crometric ASAP 2000 apparatus, while the samples were degassed at $150^{\circ} \mathrm{C}$ prior to the adsorption. Barrett-Joyner-Halenda (BJH) method was applied for the analysis of pore-size distribution, according to the adsorption isotherm. The total pore volume of the sample was estimated from the adsorbed nitrogen volume accumulated from vacuum to a relative pressure $P / P_{0}=0.95$, where $P$ is the adsorption pressure and $P_{0}$ is the nitrogen-saturated vapor pressure, which is $1 \mathrm{~atm}$ at $77 \mathrm{~K}$. The porosity of the films is defined as the ratio of pore volume to sample volume. The density of silica, $2.2 \mathrm{~g} / \mathrm{cm}^{3}$, was used for the calculation of silica volume (= weight of sample divided by silica density). To measure the various properties [refractive index $(n)$, dielectric constant $(k)$, leakage current density $(J)$, flatband voltage $\left(V_{\mathrm{FB}}\right)$, hardness $(H)$, and Young's modulus $(E)]$, the films were directly applied on various apparatuses. The refractive indices at $633 \mathrm{~nm}$ were measured by an $\mathrm{n} \& \mathrm{k}$ analyzer 1200. For capacitance and leakage current measurements, an array of alumina dots with thickness about $500 \mathrm{~nm}$ were deposited on the top side of the film by the condensation of aluminum vapor through an aluminum shadow mask. The capacitance measurements were carried out with a Keithley model $82 \mathrm{CV}$ meter. The frequency and the oscillation level were $1 \mathrm{MHz}$ and $100 \mathrm{mV}$, respectively. The dielectric constant was calculated based on the capacitance at the accumulation region of the capacitance-voltage $(\mathrm{C}-\mathrm{V})$ curve, the film thickness, and the area of electrode (which was $0.0052 \mathrm{~cm}^{2}$ ). The film thickness was measured from the crosssectional scanning by electron microscopy (SEM) taken on an S-800 (Hitachi). The flatband voltage (at which the accumulation region switched to the depletion region) was determined from the $\mathrm{C}-\mathrm{V}$ curve. For the measurement of leakage current, the back side of the silicon wafer was etched by hydrofluoric acid (HF) to remove the native oxide and was thermal-coated with aluminum. The leakage current density of the film was determined from the current-voltage (I-V) characteristics measured by an HP4156 semiconductor parameter analyzer at an electric field of $1 \mathrm{MV} / \mathrm{cm}$. The hardness and the Young's modulus of the film were measured with a Nano Indenter XP system. The value obtained near 1/10 film thickness was taken for the comparison. The Poisson ratio of the mesoporous silica film was taken as 0.25 .

\section{Results and Discussion}

By using different templates and different template/TEOS ratios, smooth films with different pore sizes were prepared. The detailed adsorption and desorption isotherms of all the samples have been presented in our previous publication. ${ }^{17}$ Moreover, XRD patterns and the porosities of the samples are shown in Fig. 1 in this paper. The pore volume, porosity, pore size, Brunauer-Emmett-Teller (BET) surface area, pore d-spacing from XRD, and estimated wall thickness of the pore of each sample are listed in Table I. Due to severe cracks after the calcination, the film templated with P123 with a weight ratio of template/TEOS $=0.41$ was not used for the later film properties measurements. The pore wall thickness was estimated by assuming that the pore in the film was in hexagonal symmetry; therefore, it was calculated from the subtraction of pore diameter (measured from nitrogen adsorption) by the value of $2 d / \sqrt{3}$, where $d$ is the pore $d$-spacing from XRD. It can be found from Fig. 1 that all the calcined films from nonionic amphiphilic templates possess XRDs which suggest the ordered pore structures. However, the ordered pore structure of the film prepared with $\mathrm{C}_{16} \mathrm{TMABr}$ (an ionic surfactant) exists before the calcination and is destroyed during the calcinations, as shown in Fig. 1a and b. Nevertheless, there are no diffraction peaks from the film prepared with PEG1450 (not a surfactant) either before or after the calcinations. Therefore, the self-assembly of the amphiphilic templates in the solution is essential to form ordered pore structures. Because various films with different pore sizes and different porosities were successfully prepared, it was possible to check whether the film properties (i.e., low- $k$, mechanical strength, and others) were correlated with the porosity. Furthermore, the porosities of the films from Tween 80 (Tween80/TEOS $=0.20$ ) and PEG1450 were within a close range. Nevertheless, the pores in the film prepared with Tween 80 were ordered in structure, while those prepared with PEG1450 were not. Thus, it is possible to investigate whether the pore ordering influences the film properties substantially.

Flatband voltage. - The flatband voltage denotes the voltage at which the accumulation region switches to the depletion region in a $\mathrm{C}-\mathrm{V}$ curve for a metal-insulator-semiconductor (MIS) unit. The value of the flatband voltage is influenced by $(i)$ the difference of the work functions of metal and semiconductor and (ii) the charges

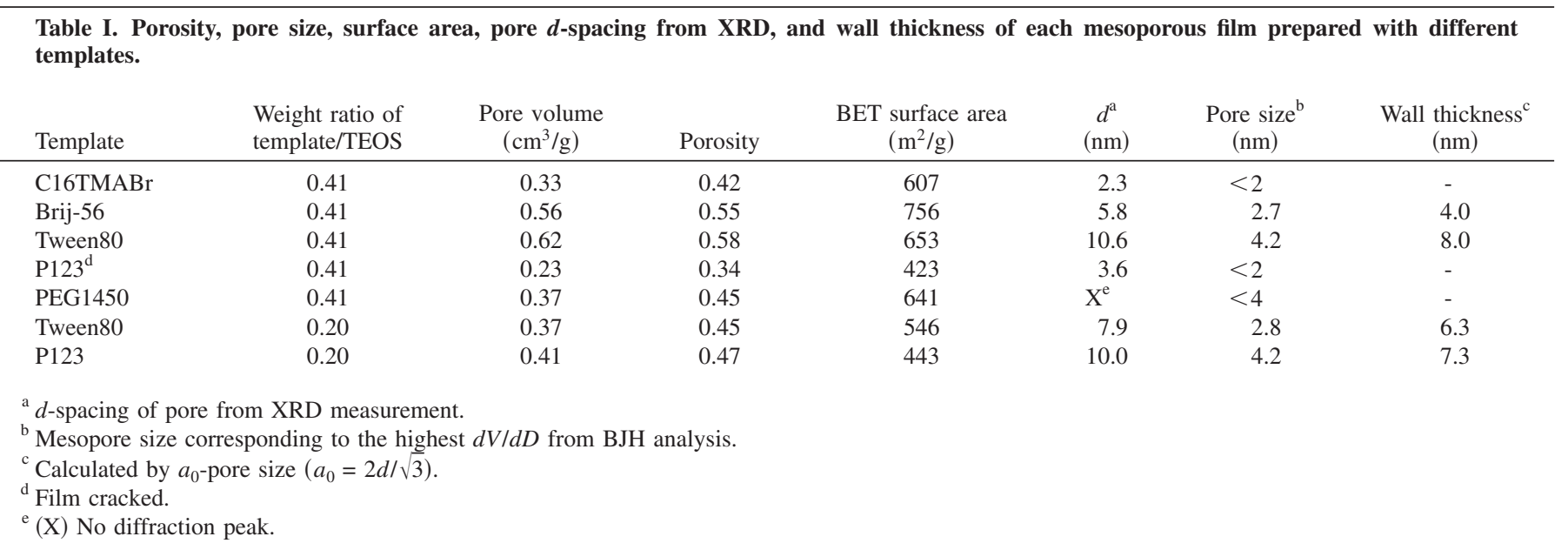




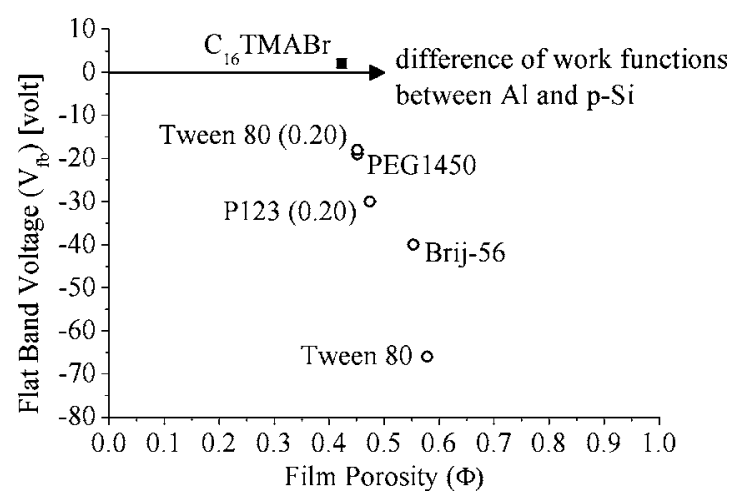

Figure 2. Correlations between flatband voltages and porosities of the films prepared with different templates. If specified, the number in the bracket after the template represents the weight ratio of template to TEOS.

within a film or at the interface of the silicon wafer and the film. ${ }^{18}$ Because the same electrode metal (aluminum) and the same semiconductor [p-type silicon $(1,0,0)$ wafer] were utilized in all the MIS units in this research, the difference of the work functions of metal and semiconductor was fixed and was in the range of -0.7 to $-1.0 \mathrm{~V}$. If there are positive charges within the film or at the interface, the flatband voltage should shift to a more negative value; in contrary, if there are negative charges, it should shift to a more positive value. However, it has been reported that the charges at the interface (also termed as interface traps) of silica are generally positive; therefore, the flatband voltages usually shift to more negative values. $^{18}$

Figure 2 shows the relationship between flatband voltages and porosities of all the samples. The flatband voltage is apparently more negative for the more porous film. This suggests that there are more positive charges either within the film or at the interface as the porosity is increased. The positive charge may be from the protons $\left(\mathrm{H}^{+}\right)$of the residual $\mathrm{Si}-\mathrm{O}-\mathrm{H}$ sites in the film frameworks. In other words, there may be more residual $\mathrm{Si}-\mathrm{O}-\mathrm{H}$ groups in the more porous film. It is noticed that the flatband voltage of the film prepared with $\mathrm{C}_{16} \mathrm{TMABr}$ surfactant is the only positive one. The positive flatband voltage indicates that there are excess negative charges within the film. The negative charges may result from the residual bromide in the framework, which is from bromine in $\mathrm{C}_{16} \mathrm{TMABr}$ after the calcination process. A trace amount of bromide is still buried in the framework even after the surface modification in the solution.

By comparing the flatband voltage of the film prepared with Tween80 (Tween80/TEOS $=0.20)$ to that prepared with PEG1450, it can be found that the two flatband voltages are approximately the same. Moreover, the porosities of these two films are also in a close range, as shown in Fig. 1. Nevertheless, the film prepared with Tween80 (Tween80/TEOS $=0.20$ ) possesses an ordered pore structure and that prepared with PEG1450 does not (as shown in XRD patterns in Fig. 1). These indicate that the porosity strongly influences the flatband voltage rather than the pore ordering or the pore structure in the film.

Leakage current.- The leakage current of the film prepared with $\mathrm{C}_{16} \mathrm{TMABr}$ (an ionic surfactant) was too large to be measured. As mentioned in the previous section, the positive flatband voltage from the film prepared with $\mathrm{C}_{16} \mathrm{TMABr}$ indicates that there were excess negative charges (possibly from bromide after the calcination process) in the film. Therefore, these negative ions may have caused the ultrahigh leakage current. It is also suggested that the films prepared with $\mathrm{C}_{16} \mathrm{TMABr}$ cannot be a qualified low- $k$ material, according to the preparation processes used in this research. It was reported in the past that the films prepared with $\mathrm{C}_{16} \mathrm{TMABr}$ were attractive as

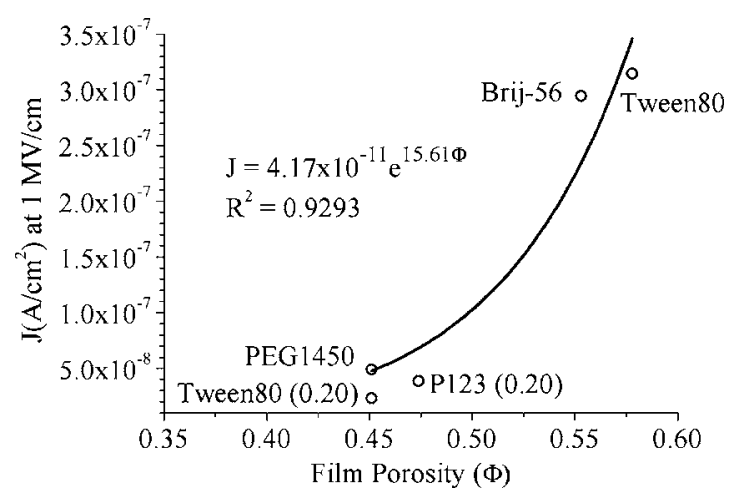

Figure 3. Correlations between leakage current densities and porosities of the films prepared with different templates. The film thickness are $344.8 \mathrm{~nm}$ from Tween80 (0.20), $406.9 \mathrm{~nm}$ from PEG1450, $425.3 \mathrm{~nm}$ from P123 (0.20), $476.7 \mathrm{~nm}$ from Brij-56, and $534.8 \mathrm{~nm}$ from Tween 80.

a low- $k$ material. ${ }^{16}$ However, the leakage current densities were not measured in that research. Therefore, their conclusions remain skeptical.

For the films prepared with nonionic templates, the relationship of the leakage current density to the film porosity is shown in Fig. 3. The leakage current density increased exponentially with the porosity. From the relationship of the flatband voltage to the film porosity shown in Fig. 2 in the previous section, it suggests that more positive charges in the more porous film. Although these charges possibly compensate the applied field, the increasing leakage current as the porosity increases indicates that the porosity plays the most dominant role on the leakage behavior. When there are more charges in the film, the combination of induced charges at the two interfaces of the insulator is easier and the leakage current density should be larger. For the films with similar porosities, for one example, by comparing the leakage current density of the film with ordered pore structure (from Tween80/TEOS $=0.20$ ) to that without (prepared with PEG1450) ordered pores, it can be found that the leakage current densities of these two films are in the same order of magnitude. For the other example, the films made with Brij-56 and Tween 80 (template/TEOS $=0.41$ ) are with 2.7 and $4.2 \mathrm{~nm}$ of different pore sizes (as listed in Table I). The leakage current densities are also in the same order of magnitude. Therefore, all of these suggest that it is the porosity substantially influencing the leakage current density of the porous films, rather than the pore size and the ordering of the pores.

Refractive index and dielectric constant.- The relationship between the refractive index and the porosity of the films and that between the dielectric constant and the porosity are shown in Fig. 4 and 5, respectively. For the films prepared with nonionic templates, both refractive index and dielectric constant decrease with the increase of porosity. Simple linear correlations can be derived and are shown in the figures. The decreases are reasonable, because the polarizability which is proportional to the refractive index and to the dielectric constant should be reduced with the decrease of the densities of the films. However, the film prepared with $\mathrm{C}_{16} \mathrm{TMABr}$ exhibited a different behavior. It can be observed in Fig. 4 that the refractive index of the film prepared with $\mathrm{C}_{16} \mathrm{TMABr}(1.428)$ deviates positively from the value (1.356) estimated from the regression line; moreover, an abnormally low $k$ value $(k=2.63)$ from the film prepared with $\mathrm{C}_{16} \mathrm{TMABr}$ can be observed in Fig. 5 . The higher refractive index implies that there are additional electronic or ionic polarizable sites in this film. These additional polarizable sites may result from the residual bromide as mentioned in the previous sections, which caused a positive flatband voltage and a very large leakage current density (out of the scale of the instrument) of the film. Moreover, the large leakage current density suggests that the induced charges at the two interfaces of the film were easily recom- 


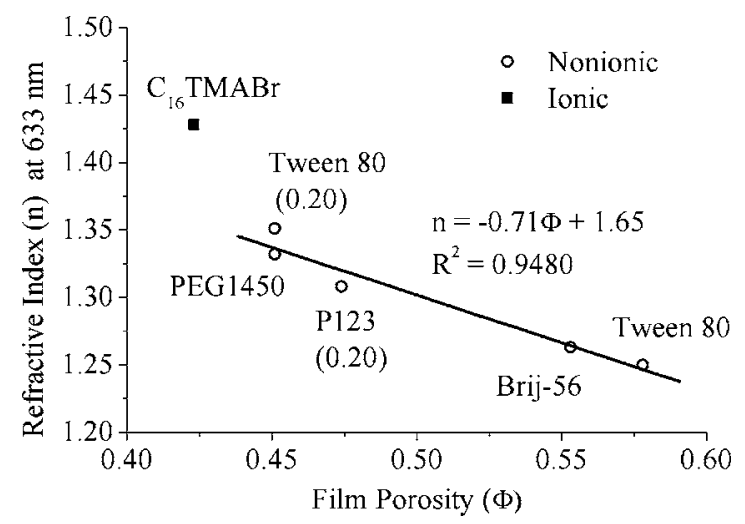

Figure 4. Correlations between refractive indices and porosities of the films prepared with different templates.

bined. During the $\mathrm{C}-\mathrm{V}$ measurement, the measured capacitance (and hence the $k$ value) was less than the intrinsic one due to the less accumulated charges at the interfaces. It may explain the abnormally low $k$ value observed from the film prepared with $\mathrm{C}_{16} \mathrm{TMABr}$. Therefore, the results in this research have shown that it is important to measure the leakage current density of the film when measuring the $k$ value, in order to judge whether the measured low value is intrinsic or not.

By comparing the refractive index of the film prepared with Tween80 (Tween80/TEOS $=0.20)$ to that prepared with PEG1450, it can be found that the refractive index of the film from Tween 80 is slightly higher than that from PEG1450. A similar trend can also be observed when the dielectric constants of these two films are compared. It suggests that there are slightly more electrical polarizable sites in the film prepared with Tween 80 (Tween80/TEOS $=0.20$ ), although the film possesses an ordered pore structure from this amphiphilic template. These electrical polarizable sites may be the residual $\mathrm{Si}-\mathrm{O}-\mathrm{H}$ sites in the frameworks. Even though the film from the amphiphilic templates possessed more polarizable sites, which can cause a higher $k$ value, more pore volume was obtained from amphiphilic templates if the same template/TEOS weight ratio is applied. In other words, the weight ratio of Tween80/TEOS $=0.20$, which was about the half of that of PEG1450/TEOS=0.41, was used for the formation of the film. Both films possessed 0.45 porosity. Because the densities of Tween80 and PEG1450 are similar, the volume of PEG1450 in the colloid solution should be twice as much as that of Tween 80 , and the pore volume of the films prepared with PEG1450 should have been substantially higher after the removal of templates. Nevertheless, the same porosity of the two films indicates that a much smaller portion of PEG1450 can form the pore volume

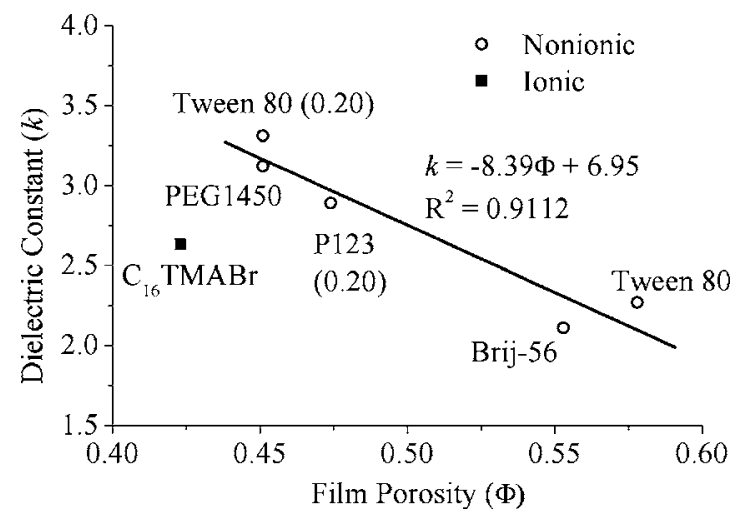

Figure 5. Correlations between dielectric constants and porosities of the films prepared with different templates.

\begin{tabular}{|c|c|c|c|}
\hline Template & Porosity & $\begin{array}{c}H / \\
(\mathrm{STD} / \mathrm{Avg}) \\
(\mathrm{GPa})\end{array}$ & $\begin{array}{c}E / \\
(\mathrm{STD} / \mathrm{Avg}) \\
(\mathrm{GPa})\end{array}$ \\
\hline $\mathrm{C}_{16} \mathrm{TMABr}^{\mathrm{a}}$ & 0.42 & $1.84 /(0.26)$ & $18.07 /(0.15)$ \\
\hline Brij-56 & 0.55 & $1.22 /(0.10)$ & $8.51 /(0.04)$ \\
\hline Tween80 & 0.58 & $1.36 /(0.11)$ & $9.18 /(0.05)$ \\
\hline PEG1450 ${ }^{a}$ & 0.45 & $1.86 /(0.20)$ & $15.42 /(0.08)$ \\
\hline Tween $80^{\mathrm{b}}$ & 0.45 & $2.52 /(0.09)$ & $25.34 /(0.05)$ \\
\hline $\mathrm{P} 123^{\mathrm{a}}$ & 0.47 & $2.12 /(0.08)$ & $18.09 /(0.15)$ \\
\hline
\end{tabular}

${ }^{\text {a }}$ Films with randomly distributed packing structures.

${ }^{\mathrm{b}}$ Weight ratio of template/TEOS is 0.2 .

in the film. Therefore, the ability of amphiphilic molecules (such as Tween80) to make more pore volume may compensate the drawback that more polarizable sites are produced via the surfactant-templated method. As shown in Table I and Fig. 5, the film with lower $k$ value (close to 2) and higher porosity (0.58) can be made by increasing the weight ratio of Tween80/TEOS to 0.41 . Moreover, the ultralow- $k$ film with $k$ value 1.47 from the colloid with Tween80/TEOS $=0.82$ has been reported previously. ${ }^{7}$

In summary, from the previous results, the flatband voltage, leakage current density, refractive index, and dielectric constant of the films made with nonionic templates have a strong correlation with the film porosity. As shown in Table I, the films with different pore sizes were prepared in this research, and the films with ordered pores and without ordered pores were also prepared. Nevertheless, both pore size and pore ordering do not substantially influence the flatband voltage, the leakage current density, the refractive index, and the dielectric constant.

Mechanical strength.- The mechanical strength of the film can be examined via the hardness and the Young's modulus (elastic modulus) of the film. The hardness represents the maximum pressure that the film can withstand. The Young's modulus represents the ability to resist the deformation. The materials are stronger if the hardness and the Young's modulus are larger. The relationship between the porosity and the mechanical strength of the films prepared in this research is summarized in Table II. It can be found that the values of the Young's modulus are all substantially larger than those reported in Ref. 16. This may be because only TEOS was used as the silica source for the preparation of coating solution in this research, instead of the mixture of TEOS and MTMS used in Ref. 16. MTMS has one methyl group directly bonded with silicon; therefore, less hydroxyl groups can be formed and available for the later condensation reactions than those from TEOS after the hydrolysis. The fewer condensation reactions may be one of the reasons for the lower values of film mechanical strength in Ref. 16.

In Table II, the data from the film templated with $\mathrm{C}_{16} \mathrm{TMABr}$ and that with PEG1450 (in which the pore structures of the films are not ordered) show that the ratios of the standard deviations to the averages of the hardness or the elastic modulus of these films are larger than those templated with nonionic surfactants (in which the pore structures of the films are ordered). This suggests that mechanical properties of the films with ordered pore structures are more uniform than those without ordered pores. Moreover, as shown in Table II, there is a trend that the hardness and the elastic modulus decrease when the film porosity increases. This is reasonable because air has replaced more silica in the more porous films. However, there is an exception. The film templated with Tween80 (Tween80/TEOS $=0.41$ ) possesses slightly stronger mechanical properties than that by Brij-56, even though the film prepared with Tween 80 is slightly more porous. It is noticed that both films have ordered pore structures. The wall thickness of the films templated with Tween 80 is thicker than that templated with Brij-56, as shown in Table I. There- 
fore, the film with a thicker pore wall certainly possesses stronger mechanical strength. Moreover, for the film templated with Tween80 (Tween80/TEOS $=0.20$ ) and that with PEG1450, the porosities are similar; the former is with ordered pore structure and the latter is without. It can be observed in Table II that the mechanical properties of the film prepared with Tween 80 are better than that with PEG1450. Thus, making a thicker pore wall and making the pore structure orderly aligned are ways to increase the film's mechanical strength.

\section{Conclusions}

In the research, the relationships between the porosity and the properties of the mesoporous silica films made with different templates have been investigated. For the films made with nonionic templates, the refractive indices and the dielectric constants decreased with the increase of porosities. They can be correlated with the porosity by simple linear functions. For the film made with ionic surfactant, $\mathrm{C}_{16} \mathrm{TMABr}$, the flatband voltage was positive. It indicates that there were excess negative charges in the film. These negative ions caused an ultrahigh leakage current. The bromide ions were a possible source of these negative ions. Therefore, nonionic templates are recommended for the preparation of low- $k$ mesoporous films in the future. For the films made with nonionic templates, the flatband voltage shifted to a more negative value when the porosity was increased. It suggests that more positive charges exist in the more porous films, which may be from the protons of silanol sites in the films. It was also found that the leakage current density increased exponentially with the increase of the porosity. The higher leakage current density may also result from the increase of silanol sites in the more porous film. Moreover, it is demonstrated in this research that the films with thicker pore walls and ordered pore structure possess better mechanical strength than those with thinner walls and without ordered pores. However, the hardness and the elastic modulus decrease with the increase of film porosity, and the porosity is the most influential factor upon mechanical strength of the film.

National Taiwan University assisted in meeting the publication costs of this article.

\section{References}

1. J. Y. Yang, C. P. Mehnert, and M. S. Wong, Angew Chem., Int Ed, 38, 56 (1999).

2. R. C. Hayward, P. Alberius-Henning, B. F. Chmelka, and G. D. Stucky, Microporous Mesoporous Mater, 44-45, 619 (2001).

3. E. L. Crepaldi, G. J. de A. A. Soler-Illia, D. Grosso, F. Cagnol, F. Ribot, and C. Sanchez, J. Am. Chem. Soc., 25, 9770 (2003).

4. S. Baskaran, J. Liu, K. Domansky, N. Kohler, and X. Li, Adv. Mater. (Weinheim Ger.), 12, 291 (2000).

5. C. Yang, A. Cho, F. Pan, T. Tsai, and K. Cho, Adv. Mater. (Weinheim, Ger.), 3, 1099 (2001).

6. C.-Y. Ting, C.-A. Wu, B.-Z. Wan, and W.-F. Wu, J. Chin. Inst. Chem. Eng., 34, 211 (2003).

7. C.-Y. Ting, D.-F. Ouyan, and B.-Z. Wan, J. Electrochem. Soc., 150, F164 (2003).

8. C.-Y. Ting, D.-F. Ouyan, W.-F. Wu, and B.-Z. Wan, Stud. Surf. Sci. Catal., 146, 391 (2003)

9. H.-T. Hsu, C.-Y. Ting, C.-Y. Mou, and B.-Z. Wan, Stud. Surf. Sci. Catal., 146, 539 (2003).

10. S.-B. Jung and H.-H. Park, Thin Solid Films, 494, 320 (2006).

11. K. Maex, M. R. Baklanov, D. Shamiryan, F. Iacopi, S. H. Brongersma, and Z. S. Yanovitskaya, J. Appl. Phys., 93, 8793 (2003).

12. Z. Wang, A. Mirta, H. Wang, L. Huang, and Y. Yan, Adv. Mater. (Weinheim, Ger.), 13, 1463 (2001).

13. S. Tanaka, J. Kaihara, N. Nishiyama, Y. Oku, Y. Egashira, and K. Ueyama, Langmuir, 20, 3780 (2004).

14. A. T. Cho, F. M. Pan, K. J. Chao, P. H. Lin, and J. Y. Chen, Thin Solid Films, 483 $283(2005)$

15. S.-B. Jung, C.-K. Han, and H.-H. Park, Appl. Surf. Sci., 244, 47 (2005).

16. F. K. de Theije, A. R. Balkenende, M. A. Verheijen, M. R. Baklanov, K. P. Mogilnikov, and Y. Furukawa, J. Phys. Chem. B, 107, 4280 (2003).

17. C.-Y. Ting, H.-S. Sheu, and B.-Z. Wan, Stud. Surf. Sci. Catal., 156, 295 (2005).

18. S. M. Sze, Semiconductor Devices, Physics and Technology, 2nd ed., John Wiley \& Sons, New York (2001). 